\title{
Conceptos para una buena toma de decisiones en la pandemia COVID-19 en Chile
}

\author{
Concepts for good decision-making in the COVID-19 pandemic in Chile
}

Mauricio Canals L. ${ }^{1}$

Programa de Salud Ambiental, Escuela de Salud Pública, Facultad de Medicina, Universidad de Chile, Santiago, Chile.

Sin financiamiento.

Ausencia de conflictos de interés.

Recibido: 29 de abril de 2020 / Aceptado: 30 de abril de 2020

L a enfermedad COVID-19 es producida por el virus SARSCoV-2, que por sus relaciones filogenéticas demuestra una clara relación con SARS-CoV-1 (agente del SARS) y por tanto con un muy probable origen en el reservorio de los murciélagos (Macroquirópteros) ${ }^{1-3}$. Los murciélagos tienen más de 76 virus con potencial patógeno humano entre los que destacan los virus Rabia, Marburg, Hendra, Nipah, Menangle, Tioman, Ébola y los coronavirus SARS y $\mathrm{MERS}^{4}$. Entonces, esta enfermedad es probablemente una zoonosis emergente a consecuencia de la trasgresión de la barrera específica, fenómeno que llamamos "spillover" o derrame.

Se le ha determinado una letalidad de 1,38\% (95\% CrI: $1,23-$ $1,53 \%)^{5-7}$, lo que no es tal alto comparando con otras enfermedades. Aunque ha causado gran cantidad de muertos, no es comparable con la cantidad de muertos que deja cada día la TBC u otras. Por ejemplo, hoy COVID-19 reporta 202597 muertos hasta el 28/4, en 3 meses $^{8}$, la influenza estacional 290.000-650.000 en un año, VIH 570.0001.100.000, Malaria 450.000, TBC 1.300.000-1.500.000 al año ${ }^{9,10}$.

El problema entonces no es su letalidad sino la gran cantidad de casos en un corto lapso de tiempo, lo que conocemos como epidemia, o pandemia si involucra a todo el mundo. Esto produce saturación de los sistemas de salud y aumenta la letalidad por falta de tratamiento adecuado, pudiendo llegar a valores muy altos como $7,7 \%$ en Italia ${ }^{11}$. Esto se complica aún más con la saturación del sistema de salud por otras enfermedades respiratorias (influenza estacional, virus respiratorio sincicial, adenovirus etc..) y produce un "efecto dominó" sobre otras enfermedades graves que necesitan unidades de tratamiento intensivo (UCI).

Así, el problema fundamental es la acelerada transmisión del virus SARS-CoV-2: la Epidemia COVID-19.

La capacidad de transmisión natural del virus se puede estimar a través del número reproductivo básico $\left(R_{0}\right)$, que se define como: el número de casos nuevos producidos en promedio por cada caso, en un intervalo serial, en una población completamente susceptible. El intervalo serial $(\tau)$ corresponde al promedio de tiempo que pasa entre contagio y contagio y es equivalente al concepto de tiempo generacional de una población (por ejemplo, la esperanza de vida del hombre es de alrededor de 80 años y su tiempo generacional es de 30 años, del mismo modo que la esperanza de vida infectante de COVID-19 es 14 días, pero su intervalo serial es $\tau=5$ días). Aunque hay controversia al respecto ${ }^{12}$ un valor aproximado y razonable para COVID-19 es $R_{0}=2,35$.

Durante una epidemia, el número de susceptibles va decreciendo, y a consecuencia de ello, la probabilidad de transmisión va decreciendo hasta que en algún instante se produce el efecto conocido como inmunidad comunitaria o de "rebaño" ("herd immunity") que hace que la transmisión se desacelere y finalmente se detenga; lo que John Brownlee (1868-1927) llamaba "la dilución de los susceptibles en los

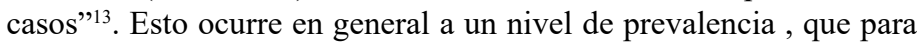
el caso de COVID-19 es aproximadamente $57,4 \%$ para $R_{0}=2,35^{14}$. Durante las epidemias, además se toman medidas epidemiológicas y de control de la enfermedad, haciendo que el $R_{0}$ sea una inadecuada medida de transmisión durante la epidemia, por lo que es mejor el concepto de número reproductivo efectivo, que varía en el tiempo, $R_{e}(t)$, que corresponde al mismo concepto que $R_{0}$, pero bajo los efectos de las medidas de control y mitigación durante la epidemia.

Durante una epidemia se debe evitar la sobrecarga de los servicios de salud y reducir la transmisión $(\operatorname{Re}(t))$.

Ya que los pacientes más graves necesitan ser internados en UCI, una medida adecuada para medir la sobrecarga es comparar la disponibilidad de UCI, con una estimación de la necesidad de UCIs en un tiempo determinado. Esto se puede estimar de dos formas:

a) Máxima tolerancia diaria de pacientes (MTD): si tengo una disponibilidad de 583 UCIs (con ventilador) (28/4) y cada UCI es usada en promedio 14 días ${ }^{(5-7)}$ entonces el sistema sólo tolerará $583 / 14=42$ pacientes por día. Como $5 \%$ de los casos requiere UCI, el sistema tiene una máxima tolerancia diaria de MTD $=42 / 0.05=840$ casos por día.

b) Carga esperada de UCI (E(UCI)): Como cada infectado activo (I(t)) deja en promedio $R_{e}(t)$ nuevos casos en un intervalo serial $(\tau)$; el 
número de pacientes nuevos que se producen en un intervalo serial se puede calcular multiplicando el número de infectados "activos" (infectados infectantes $(I(t))$ por $R_{e}(t)$. Los infectados activos se puedes estimar como los casos nuevos acumulados en las dos últimas semanas (14 días). Un 5\% de ellos necesitará UCI con un retardo de una semana ${ }^{5,6}$. El 28/4 se tienen 6.448 infectados activos y $R e=1,1$ (cálculo personal). Entonces, se generarán $6.448 \times 1,1=7.093$ nuevos casos en 5 días $(\tau)$ y $5 \%=0,05 \times 7.093=355$ necesitarán UCI en las próximas dos semanas, ya que hay un retardo en la necesidad de UCI de 7 días $^{5,6}$. Este valor lo podemos comparar con la disponibilidad de UCIs con ventilador disponibles.

El número reproductivo efectivo $R_{e}(t)=q(t) R_{0} \mathrm{y} q(t)$ representa la proporción de susceptibles en el tiempo ${ }^{15}$. Esta se va reduciendo a lo largo de la epidemia en forma natural por el "efecto rebaño". Pero a menos que se tenga una vacuna, es un proceso muy largo y necesariamente tendría como consecuencia gran cantidad de muertos y saturación de los sistemas de salud. Entonces hay que acelerar el proceso disminuyendo $R_{0}$. Este se puede expresar como:

$$
\mathrm{R}_{0}=\frac{\beta X o}{(\gamma+\mu)}
$$

donde $\beta$ es el coeficiente de transmisión, Xo es la densidad inicial de susceptibles, $\gamma$ es la tasa de recuperación y $\mu$ la tasa de mortalidad. Es decir $R_{0}$ es el producto del potencial reproductivo $(\beta X o)$ por la esperanza de vida infectante $(1 /(\gamma+\mu))^{16}$. La reducción de $R_{0}$ se puede lograr aumentando la tasa de recuperación, $\gamma$, es decir encontrando un adecuado tratamiento, lo que hasta ahora no ha resultado posible. Entonces el objetivo principal de la mitigación es disminuir el coeficiente de transmisión ( $\beta$ : transmissibility). Este lo podemos descomponer en: $\beta=b P(I / C) P(C)$, donde $b$ es la tasa de contacto entre las personas, $P(C)$, la probabilidad de contacto infectante y $P(I / C)$ es la probabilidad que un contacto infectante tenga como resultado una infección ${ }^{17,18}$.

Las intervenciones epidemiológicas deben ir dirigidas a la disminución de uno o más de estos tres factores:

a) $P(I / C)$ Probabilidad de que un contacto infectante resulte en infección: uso de mascarillas, medidas de higiene personal, uso de desinfectantes como alcoholgel, lavado de manos, vacuna.

b) $b$ Tasa de contacto: medidas de inmovilidad, desagregación y distanciamiento social.

c) $P(C)$ Probabilidad de contacto infectante: trazabilidad y aislamiento de infectados y contactos, cierre de colegios y universidades, cuarentenas, cordones sanitarios $\mathrm{y}$ cierre de fronteras.

Estas intervenciones han logrado en Chile reducir un $R_{0}$ de 2,38 a valores de $R_{e}(t)$ actuales de 1,1 (cálculos personales). Pero hay que hacer notar que mientras no exista una vacuna (que afecta directamente y dramáticamente $P(I / C)$ y $q(t)$ ), el virus conserva la capacidad potencial de volver a valores cercanos a $R_{0}$ cuando se relajen las intervenciones epidemiológicas, ya que el efecto rebaño aún no parece haberse producido. El des-escalamiento de las intervenciones debiera considerar cuatro aspectos al menos: Carga de infectados activos $(I(t))$, número reproductivo efectivo $\left(R_{e}(t)\right)$, carga del sistema de salud (CS: carga UCI y personal de salud) y esfuerzo diagnóstico (ED), que es el que permite la identificación de casos, su trazabilidad y aislamiento. En términos cualitativos, se podría estimar el riesgo $(R g)$ del des-escalamiento de las intervenciones como:

$$
\operatorname{Rg} \alpha=\frac{I(t) \operatorname{Re}(t) C S}{E D} ;
$$

es decir, el levantamiento de las medidas será mas riesgoso mientras mas carga de infectados activos, mientras mas alto el número reproductivo efectivo y mientras mayor sea la carga sobre el sistema de salud. El riesgo será menor mientras mayor sea el esfuerzo diagnóstico y trazabilidad asociada.

\section{Referencias bibliográficas}

1.- Andersen K V, Rambaut A, Lipkin W I, Holmes E C, Garry R F. The proximal origin of SARSCoV-2. Nature Medicine. 2020; 26: 450-5. https://doi.org/10.1038/s41591-020-0820-9.

2.- Zhang Y Z, Holmes E C. A genomic perspective on the origin and emergence of SARS-CoV-2. Cell. 2020. doi: https://doi. org/10.1016/j.cell.2020.03.035

3.- $\quad$ Lam T T Y, Shum M H, Zhu H C, Tong Y G, Ni $\mathrm{X}$ B, Liao Y S, et al. Identifying SARS-CoV-2 related coronaviruses in Malayan pangolins. Nature. 2020. doi: 10.1038/s41586-020-2169-0

4.- Callisher C H, Childs J E, Field H E, Holmes K
V, Schountz T. Bats: important reservoir hosts of emerging viruses. Clin Microbiol Rev 2006; 19 (3): 531-45. doi:10.1128/ CMR.00017-06

5.- Russell T W, Hellewell J, Jarvis CI, van Zandvoort K, Abbott S, Ratnayake R, et al. Estimating the infection and case fatality ratio for coronavirus disease (COVID-19) using age-adjusted data from the outbreak on the Diamond Princess cruise ship, February 2020. Euro Surveill 2020 Mar; 25(12): 2000256. doi: 10.2807/1560-7917. ES.2020.25.12.2000256.

6.- Russell T, Hellewell J, Abbott S, Golding N, Gibbs H, Jarvis CI, et al. Using a delay- adjusted case fatality ratio to estimate underreporting. CMMID; London School of Hygiene \& Tropical Medicine. CMMID Repository. April 2020; cmmid.github.io/topics/covid19/ global_crf_estimates.html.

7.- Guan W, Ni Z, Hu Y, Liang W, Ou C, He J, et al. Clinical characteristics of Corona disease. 2019 in China. N Engl J Med March 6, 2020, at NEJM.org. doi: 10.1056/NEJMoa2002032.

8.- WHO Coronavirus disease (COVID-2019) situation reports. Situation report 99.https:// www.who.int/emergencies/diseases/novelcoronavirus-2019/situation-reports/

9.- WHO. World health report. Infectious diseases kill over 17 million people a year: WHO warns 
of global crisis. https://www.who.int/whr/1996/ media_centre/press_release/en/ (consultado en Abril 2020).

10.- Roth G A, Abate D, Abate K H, Abay S M, Abbafati C, Abbasi N, et al. Global, regional, and national age-sex-specific mortality for 282 causes of death in 195 countries and territories, 1980-2017: a systematic analysis for the Global Burden of Disease Study 2017. The Lancet, 2018; 392(10159): 1736-88. doi: 10.1016/ S0140-6736(18)32203-7

11.- Lazzerini M, Putoto G. COVID-19 in Italy: momentous decisions and many uncertainties. Lancet Glob Health 2020; https://doi. org/10.1016/S2214-109X(20)30110-8.

12.- Sanche S, Lin Y T, Xu C, Romero-Severson
E, Hengartner N, Ke R. High contagiousness and rapid spread of Severe Acute Respiratory Syndrome Coronavirus 2. Emerg Infect Dis. 2020 Jul 4. https://doi.org/103201/ eid2607.200282

13.- Fine P E M. John Brownlee and the measurement of infectiousness: an historical study in epidemic theory. J. R. Statist. Soc A 1979; 142 (3): 347-62. https://doi. org/10.2307/2982487

14.- Anderson RM. How will country-based mitigation measures influence the course of the COVID-19 epidemic? The Lancet.com Vol 395 March 21, 2020 Published Online March 6, 2020 https://doi.org/10.1016/ S01406736(20)305675
15.- Chowell G, Hyman J M, Bettencourt L M A, Castillo-Chávez C. Mathematical and statistical estimation approaches in epidemiology. Heidelberg: Springer; 2009.

16.- Anderson RM. Epidemiology. In Modern Parasitology (Cox FEG ed.). Oxford: Blacwell Scientific Publications; 1993. 75-117pp.

17.- Canals M., Bustamante RO, Ehrenfeld M., \& Cattan P.E. 1999. Assesing the impact of insect vectors on animal populations. Acta Biotheoretica 2018 (Dec); 46: 337-45. doi: 10.1023/A:1001891017457

18.- Canals M. Introducción a la epidemiología matemática. Santiago: Edición Sociedad Chilena de Parasitología, Chile ISBN: 978-956368-734-7; 2017. 